Z. Yin, I. Boxx, and W. Meier, Influence of self-sustained jet oscillation on a confined turbulent flame near lean blow-out, Proceedings of the Combustion Institute 36 (2017) 37733781

The original publication is available at www.elsevier.com

http://dx.doi.org/10.1016/j.proci.2016.07.026 
Title: Influence of self-sustained jet oscillation on a confined turbulent flame near lean blow-out

Authors: Zhiyao Yin, Isaac Boxx, Wolfgang Meier

Affiliation: Institute of Combustion Technology, German Aerospace Center (DLR), 70569, Stuttgart, Germany

Corresponding author: Zhiyao Yin (Email: zhiyao.yin@dlr.de), B239, Pfaffenwaldring 38-40, 70569, Stuttgart

Colloquium: Gas Turbine Combustion

Word Count: $5910($ Method I)

$\begin{array}{cccc}\text { Main Text: } & & & 4005 \\ \text { References: } & & & 385 \\ \text { Equations: } & & & 69 \\ & & \text { Figure size } & \text { Caption } \\ & & & \\ \text { Fig.1: } & 180 & 20 & 200 \\ \text { Fig.2: } & 73 & 9 & 82 \\ \text { Fig.3: } & 191 & 27 & 218 \\ \text { Fig.4: } & 227 & 21 & 248 \\ \text { Fig.5: } & 150 & 10 & 160 \\ \text { Fig.6: } & 356 & 21 & 377 \\ \text { Fig.7: } & 152 & 14 & 166 \\ \text { Figures: } & & & 1451 \\ \text { Total } & & & \mathbf{5 9 1 0}\end{array}$




\section{List of figures}

Figure 1 (a) Schematics of the bottom section of combustor chamber (b-d) average flow field, $\mathrm{OH}$ and $\mathrm{OH}^{*}$ signal distributions

Figure 2 Observed flame fluctuations with long duty cycle

Figure 3 (a) Energy contributions of the POD modes and the first spatial mode for the (b) velocity field, (c) $\mathrm{OH}$ and (d) $\mathrm{OH}^{*}$ signal distributions.

Figure 4 Phase modeling of the velocity field and $\mathrm{OH}^{*}$ signal distribution based on the spatial modes given in Fig.3

Figure 5 A time segment containing a flame "puffing" event

Figure 6 Top to bottom: a sequence of the instantaneous flow fields and $\mathrm{OH}^{*}$ distributions detailing a flame puffing event

Figure 7 Statistical mapping of (a) $\left\langle\mathrm{OH}^{*}\right\rangle$ and (b-c) partial blow-out/re-light events 


\title{
Influence of self-sustained jet oscillation on a confined turbulent flame near lean blow-out
}

\author{
Zhiyao Yin ${ }^{\mathrm{a}, *}$, Isaac Boxx ${ }^{\mathrm{a}}$, Wolfgang Meier ${ }^{\mathrm{a}}$ \\ ${ }^{a}$ Institute of Combustion Technology, German Aerospace Center, 70569, Stuttgart, Germany
}

\begin{abstract}
Premixed methane-air turbulent flame is generated in a single-nozzle jet-stabilized combustor designed based on the FLOX ${ }^{\circledR}$ concept ${ }^{1}$. Confinement-induced, self-sustained jet oscillation is observed. Its influence on combustion stability near lean blow-out (LBO) is investigated using simultaneous particle imaging velocimetry (PIV), planar laser-induced fluorescence of $\mathrm{OH}$ radicals $(\mathrm{OH}$ PLIF), and $\mathrm{OH}$ chemiluminescence imaging at 5-kHz repetition rate. Via proper orthogonal decomposition (POD) of the velocity field and extended POD of the scalar fields, pronounced variations in the flame shape are observed during a cycle of jet oscillation. In extreme cases, flame is partially blown out in the combustor due to jet impingement on the wall during the first half of its oscillation cycle. In the subsequent half cycle following jet detachment, flame is restabilized after robust flashback and re-light. Statistical analysis shows that such pattern is by far the most prevalent mechanism for blow-out and restabilization to take place at the operating condition. Additionally, these events are found with much higher probability during slow-paced jet oscillations.

Keywords: Confined Turbulent Flames, Jet Oscillation, Highspeed Laser Diagnostics, Proper Orthogonal Decomposition
\end{abstract}

\section{Introduction}

FLOX $^{\circledR}$ combustion, also termed as flameless [1] or mild combustion [2], has recently gathered great interest for its low susceptibility to thermoacoustics and flashback [3] as well as its high fuel flexibility and low NOx emission [4]. It is often regarded as a viable alternative to swirl-stabilized flames for stationary gas turbines. FLOX $^{\circledR}$ combustors generally consist of circularly arranged

\footnotetext{
*Corresponding author

Email address: zhiyao.yin@dlr.de (Zhiyao Yin)

${ }^{1} \mathrm{FLOX}^{\circledR}$ is a registered trademark of WS Wärmeprozesstechnik GmbH, Renningen, Germany
} 
nozzles issuing high momentum jets of reactants into a combustion chamber, generating strong flow recirculation and hence intense mixing of reactants and products. Characterization of various FLOX ${ }^{\circledR}$ combustors is an ongoing effort at our institute, with early investigations dedicated to obtaining quantitative data sets for validating numerical simulations [4, 5, 6]. With a recent focus on the stabilization mechanisms in this type of combustors, a laboratory-scale single-nozzle FLOX ${ }^{\circledR}$ combustor has been designed to allow more sophisticated optical diagnostics [5]. Subsequently, confinement-induced periodic jet oscillation has been identified as the major source of instability in flames stablized in this combustor [7, 8].

Confinement-induced, self-excited and self-sustained jet oscillation is a well-documented phenomenon in non-reacting flows [9]. It is commonly understood that, the oscillation is triggered by obstructed shear layers (such as by a recirculation zone), and is sustained by a feedback loop between initial disturbances and the impinging points. Such feedback is most commonly hydrodynamic, as seen in jets experiencing sudden expansion [10, 11] and jets issuing into a cavity [12, 13]. Jet flapping and jet precession have been identified as the primary patterns of oscillation in confined jets [10, 11, 12]. The former case is often found in planar jets, where recirculation zones on each side of the jet move conversely upstream or downstream as the jet flaps with respect to the plane of symmetry. Through proper orthogonal decomposition (POD) of the 2-D velocity field [14], the spatial mode responsible for the jet flapping was found to contain large structures aligned along each side of the jet. In the case of a round jet expanding into a concentric cylindrical chamber [10, 15], jet precessing was found to induce a swirling flow in the confinement. Through parametric studies, oscillations in confined jets have been found to scale linearly with jet velocity and impingement length (e.g., nozzle diameter and confinement dimensions) [12, 13]. They often possess a Strouhal number in the range of St 0.001-0.01, much smaller than the instabilities generated within shear layers $(S t \sim 1)$ [10]. As its main influence on the flow field, jet oscillation can drastically increase jet spreading rate and enhance large scale entrainment of the ambient fluid while suppressing fine scale mixing [11]. In reacting flows, these features were found responsible for an increased flame volume and a subsequent reduction in NOx production, compared with flames supported by non-oscillating jets [16].

Despite enduring interest in jet oscillation in various research fields, its implications on com- 


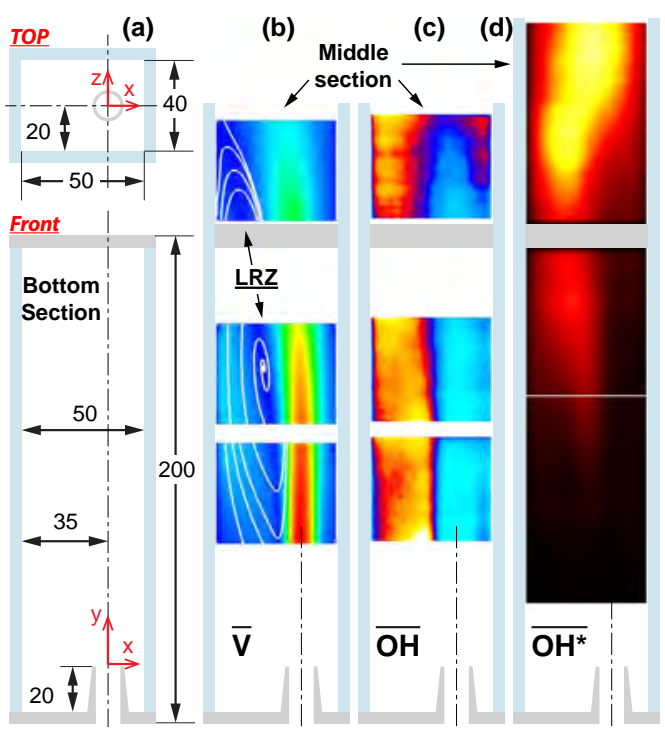

Figure 1: (a) Schematics of the bottom section of combustor chamber (b-d) average flow field, $\mathrm{OH}$ and $\mathrm{OH}^{*}$ signal distributions

bustion stability have not been fully explored. With the development of the single-nozzle FLOX ${ }^{\circledR}$ combustor mentioned above, we recently reported on the complex pattern of jet flapping and its various influence on flame structure and flame stabilization when operating at stable conditions [8]. Since FLOX ${ }^{\circledR}$ combustors typically operate at fuel-lean conditions, mechanisms controlling the lean blow-out (LBO) process are of particular interest for extending their operational limits. Operating at a specific condition where flame blow-out occurs partially and sporadically, the current work examines the relationship between jet oscillation and the LBO mechanisms in the single-nozzle FLOX ${ }^{\circledR}$ combustor. To time-resolve the transient combustion dynamics at this condition, fluctuations in the flow field as well as the scalar fields are measured using 5-kHz-rate, simultaneous stereo particle imaging velocimetry (PIV), planar laser-induced fluorescence of OH radicals (OH PLIF) and $\mathrm{OH}$ chemiluminescence imaging.

\section{Experimental}

\subsection{Combustor and operating conditions}

The single-nozzle, single-channel combustor consisted of three identical sections stacked on top of each other. Each of these sections was 200-mm tall, had a cross section of $50 \mathrm{~mm}$ by $40 \mathrm{~mm}$, and was enclosed by quartz windows to provide four-way, wall-to-wall clear optical access. The bottom section is illustrated in Fig:1 1 . The entire combustor chamber was mounted on a three-axis 
translation stage, to allow repositioning relative to the diagnostic setup. Premixed methane-air mixture was delivered through a straight stainless steel tube (ID=10 mm, L=400 $\mathrm{mm}$ ) into the combustor. The nozzle had a chamfered tip, rose $20 \mathrm{~mm}$ from the base plate, and was offset by 10 $\mathrm{mm}$ along the $\mathrm{x}$-axis from the geometric center of the combustor chamber. The off-center positioning of the jet was designed to draw analogy to the situation around a jet nozzle in an actual FLOX ${ }^{\circledR}$ combustor [5]. The coordinate system used in this work is defined in Fig,1].

For this study, the combustor was operated with a jet exit velocity of $63 \mathrm{~m} / \mathrm{s}$, at initial temperature of $300 \mathrm{~K}$ and pressure of 1 bar. Methane and air were well mixed in a static mixer three meters upstream of the jet exit. During a measurement, flame was first stabilized initially at $\phi=0.8$. Once the combustor was thermally stable, the methane flow rate was gradually reduced to reach a stoichiometry of $\phi=0.77$, which was close to the lean blow-out (LBO) limit of about $\phi=0.75$. At this condition, flame becomes unstable and undergoes sporadic processes of partial blow-out and re-light.

\subsection{5-kHz-rate diagnostic setup}

The stereo PIV system utilized a dual-cavity Nd:YAG laser (Edgewave IS-6IIDE) and a pair of CMOS cameras (LaVision HSS8) equipped with $\mathrm{f}=200 \mathrm{~mm}$ lenses (f/5.6). The two cameras were coupled with Scheimpflug adaptors, suspended on opposite sides of $\mathrm{x}-\mathrm{y}$ plane of the combustor, focused down onto the same field of view (FOV). For OH PLIF, a frequency-doubled dye laser (Sirah Credo) was pumped by an Nd:YAG laser (Edgewave IS-8IIE). The laser system was tuned to the peak of $\mathrm{Q}_{1}(7)$ line in the $\mathrm{OH} \mathrm{A}-\mathrm{X}(1,0)$ band. OH fluorescence was collected into an intensified camera system (Lavision HS-IRO and HSS6 CMOS) through a UV lens (Halle , f=100 mm, f/2.8, coupled with a 310-nm bandpass filter). The OH PLIF excitation pulse was temporally placed between the two PIV pulses. Both laser sheets were overlapped and aligned through the center of the nozzle (x-y plane). A common FOV of about $50 \times 35 \mathrm{~mm}^{2}$ (in the front view) was achieved

for both measurements (which covers the entire chamber width). Based on \pm 0.1 pixel uncertainty of the cross correlation peak-finding algorithm and the $\Delta t=6 \mu$ s set between the two PIV laser pulses, an uncertainty of $\pm 0.8 \mathrm{~m} / \mathrm{s}$ was estimated for the velocity measurement. For $\mathrm{OH}^{*}$ imaging, a filtered (310-nm bandpass) UV lens (Cerco, $\mathrm{f}=45 \mathrm{~mm}, \mathrm{f} / 1.8$ ) with a set of HS-IRO and CMOS 


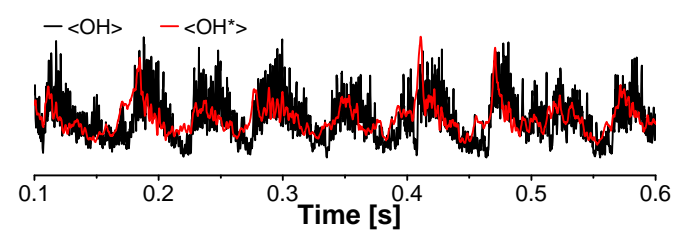

Figure 2: Observed flame fluctuations with long duty cycle

camera (Lavision HSS5) was used to monitor the global flame behavior from the front view (with a FOV of about $250 \mathrm{~mm}$ tall). The camera gate was set to open $0.5 \mu \mathrm{s}$ after the PLIF laser pulse to avoid interference from $\mathrm{OH}$ fluorescence. Note that the camera systems for OH PLIF and $\mathrm{OH}^{*}$ were set perpendicular to and on opposite sides of the x-y plane.

\section{Jet-oscillation-induced combustion instabilities}

\subsection{General observations}

General flow field and flame shape at the current operating condition were found similar to results in other premixed methane-air flames generated in the same combustor [5, 7, 8]. Fig,1b-d shows respectively the average flow field, $\mathrm{OH}$ and $\mathrm{OH}^{*}$ signal distributions, from measurements conducted at different combustor positions along the y-axis. Combustion instabilities were first observed in spatially integrated $\mathrm{OH}$ LIF and $\mathrm{OH}^{*}$ signal in the middle section of the combustor chamber (where $\mathrm{OH}^{*}$ is the strongest). An example from one set of high-speed measurements is given in Fig.2. Both $\langle\mathrm{OH}\rangle$ and $\left\langle\mathrm{OH}^{*}\right\rangle$ (angle brackets denote spatial integration) exhibit distinctive and periodic fluctuations with nearly identical duty cycle. Such long duty cycle, at about $f=20 \mathrm{~Hz}$ or $S t_{d}=0.003$, is consistent with the characteristic frequencies of jet oscillation at stable operating conditions reported in Refs. [7, 8]. In addition to such periodic fluctuations, sporadic flame "puffing" resembling an incomplete flame blow-out was observed during the measurements. From high-speed recordings of $\mathrm{OH}^{*}$ images, the nature of these events was identified as a partial blow-out followed by flame restabilization in the bottom section of the combustor.

To confirm the correlation between jet oscillation and the observed combustion instabilities, a series of analyses were carried out based on the POD technique utilizing the time-resolved, simultaneous measurements of velocity and scalar fields. A step-by-step demonstration is provided in the following sections. 


\subsection{Basics of POD and its extension}

POD is a well-established tool for extracting and characterizing coherent structures in unsteady flow fields [17]. Briefly, POD identifies an orthonormal basis, formed by POD modes $\boldsymbol{\phi}_{j}$, to optimally represent the fluctuating portion of the velocity field $\boldsymbol{u}^{\prime}$, so that the instantaneous flow field can be reconstructed using (terminology following Ref.[18]):

$$
\boldsymbol{u}_{i}=\overline{\boldsymbol{u}}+\sum_{j=1}^{N} a_{i j} \cdot \boldsymbol{\phi}_{j}
$$

where $\overline{\boldsymbol{u}}$ is the average velocity field, $a_{i j}=\left\langle\boldsymbol{u}^{\prime} \cdot \boldsymbol{\phi}_{j}\right\rangle$ (angle bracket denotes spatial integration) is known as the temporal coefficient of mode $j$. In this work, the snap-shot method [19] was used to derive $a_{i j}$ by solving the Eigenvalue problem of the correlation matrix $K_{i j}=\left\langle\boldsymbol{u}_{i}^{\prime} \cdot \boldsymbol{u}_{j}^{\prime}\right\rangle$, constructed from $N$ snap shots (see details in Ref.[18]). Then, the spatial modes were obtained by:

$$
\phi_{j}=\frac{1}{\lambda_{j} N} \sum_{i=1}^{N} a_{i j} \cdot \boldsymbol{u}_{j}^{\prime}
$$

where $\lambda_{j}$ is the Eigenvalue associated with mode $j$, which also represents the contribution of mode $j$ to the total kinetic energy.

In a similar manner, the so-called "extended" POD modes [20] can be derived for the simultaneously measured scalar fields $\left(\mathrm{OH}\right.$ and $\mathrm{OH}^{*}$ in this case). The extended modes $\chi_{j}$ of a given scalar field $c_{j}$ can be calculated based on $a_{i j}$ from POD of the flow field, and by replacing $\boldsymbol{u}_{j}^{\prime}$ with the fluctuating part of the scalar field $c_{j}^{\prime}$ in Eq. (2). In essence, scalar field fluctuations dictated by $\chi_{j}$ is by definition a direct result of the flow field oscillation described by $\phi_{j}$.

\subsection{Spatial modes}

Figure 3b-d shows respectively the first POD mode and its extended modes of the velocity field, $\mathrm{OH}$ and $\mathrm{OH}^{*}$ signal distributions, obtained from decomposing sets of 3500-shot measurements taken at different combustor locations (marked by numbers in parenthesis). From Fig. $3 b$, the first POD mode (termed the "flapping mode" in this work) comprises islands of velocity peaks (refer to the magnitude of $\mathrm{Vy}$ in Fig[3b) distributed on each side of the jet, typical of a flapping jet [14]. Also, the flapping mode is by far the most dominant flow structure at the current condition. This is evident 


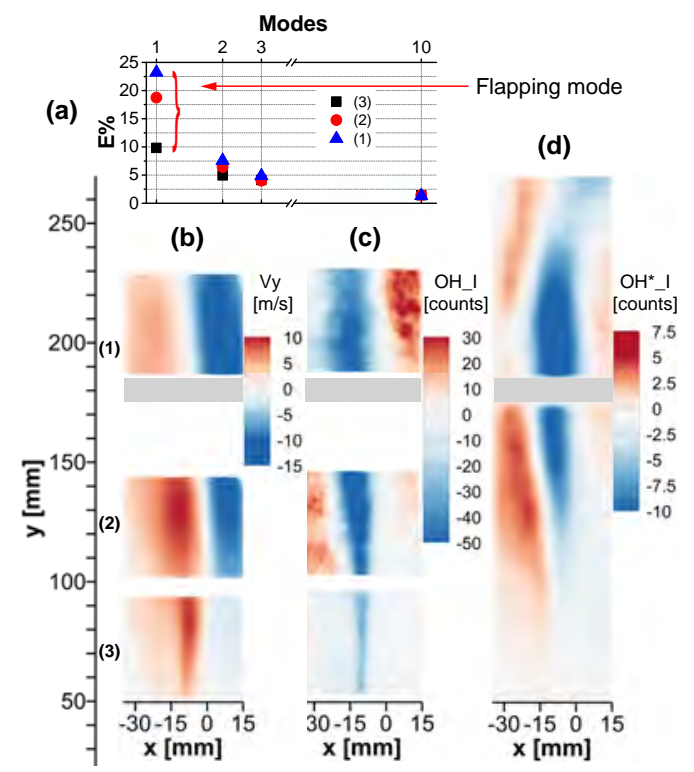

Figure 3: (a) Energy contributions of the POD modes and the first spatial mode for the (b) velocity field, (c) $\mathrm{OH}$ and (d) $\mathrm{OH}^{*}$ signal distributions.

from its significantly higher energy contribution compared to the rest of the modes at all three measurement locations, especially in the middle section of the chamber, as is shown in Fig 3 a. This feature is rather unique to this combustor owing to the asymmetric setting of the jet. In symmetric confinements, the first two modes normally contribute similarly to the total energy [14, 15]. On the other hand, the extended modes of $\mathrm{OH}$ and $\mathrm{OH}^{*}$ distribution (Fig.3 3 -d) are quite similar, despite the latter being a line-of-sight integration. Both consist of negative intensity regions near the jet axis sandwiched by positive regions near the walls.

\subsection{Low-order modeling of the oscillating fields}

The interpretation of the spatial modes in Fig 3 and their relations to jet flapping can be better comprehended through a phase $(\psi)$-dependent low-order modeling [18]. This is done by using the obtained spatial modes as a basis and assuming a sinusoidal jet oscillation. The phase-dependent fields can therefore be modeled as:

$$
\boldsymbol{f}(\psi) \approx \overline{\boldsymbol{f}}+\sqrt{\lambda_{F}} \cdot \sin \psi \cdot \boldsymbol{e}_{F}
$$

where $\boldsymbol{f}(\psi)$ represents either the velocity or the scalar field, $\bar{f}$ is the time average, $\lambda_{F}$ is the Eigenvalue of the flapping mode, and $\boldsymbol{e}_{F}$ denotes the flapping mode $\boldsymbol{\phi}_{F}$ or its extended modes 


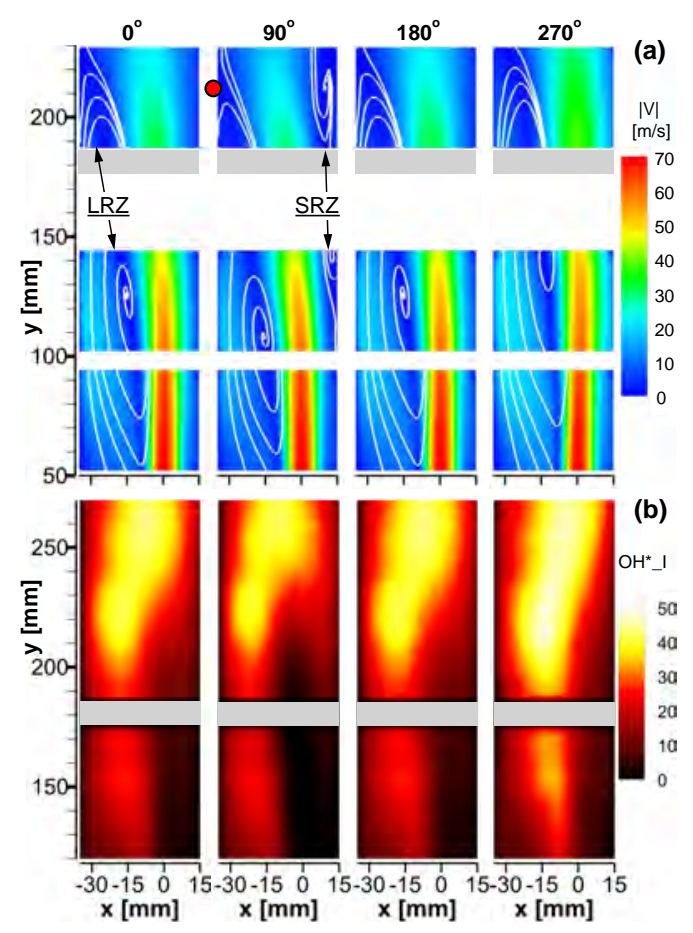

Figure 4: Phase modeling of the velocity field and $\mathrm{OH}^{*}$ signal distribution based on the spatial modes given in Fig 3

$\chi_{F}$. Note that although there is a coupling between the flapping mode and higher order modes [8], Eq.(3) is a valid approximation by neglecting the higher order modes due to their much lower energy contributions (2-4 times lower than the flapping mode, see Fig.3a).

Figure 4 shows the modeled fields at four representative phases depicting an oscillating cycle. The same sets of data shown in Fig 3 were used. From Fig, 4a, in the first half cycle $\left(0^{\circ}\right.$ to $\left.180^{\circ}\right)$, the jet flaps left then right, causing the impingement point (red mark) and the LRZ to shift upstream and downstream. Concurrently to the opposite side of the LRZ, a secondary recirculation zone (SRZ) forms and disappears. Although it is a transient structure at the current condition, the SRZ could also be a permanent fixture in the flow field, as found at other operating conditions in Ref. [8]. In the next half cycle $\left(180^{\circ}\right.$ to $\left.360^{\circ} / 0^{\circ}\right)$, the jet flaps right then left, during which the jet almost straightens up against the right wall, with a notable increase of velocity in the jet stream.

From Fig $4 \mathrm{~b}$ of phase-dependent $\mathrm{OH}^{*}$ distributions, flame contracts and retreats downstream as the jet flaps leftwards. The trend is reversed during the second half of the cycle. Flame stretches along the y-direction, attaining a much larger volume and higher intensity at phase $270^{\circ}$ than at $90^{\circ}$. Consistent with the $\mathrm{OH}^{*}$ fluctuations, it was also observed that the oscillation of LRZ following jet flapping causes the region containing $\mathrm{OH}$ signal (mostly in the LRZ) to narrow and widen. 


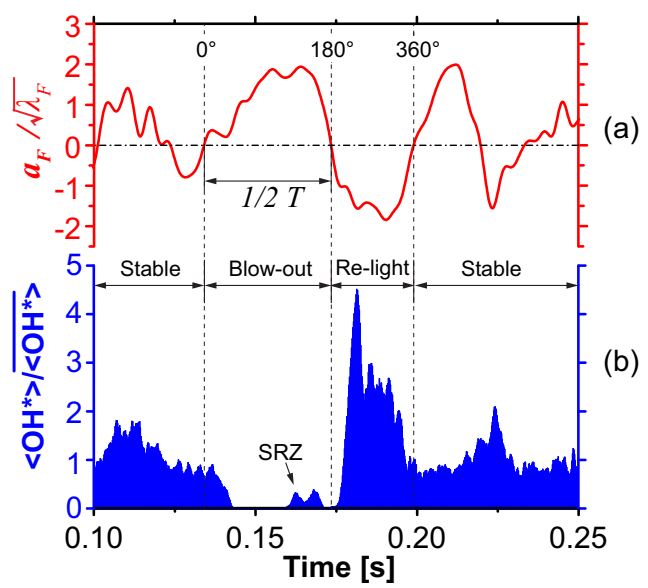

Figure 5: A time segment containing a flame "puffing" event

Alternatively to the low-order modeling method, phase averaging can be performed using the temporal coefficient $a$. Ref.[18] compared the two techniques in great detail, finding only minor differences in their phase-sorted results. Phase averaging was however deemed less suitable for the current work, mainly because the slow jet flapping motion requires large sets of runs for convergence at a given phase. It may also smear out important features due to the multi-frequency nature of the flapping motion [8].

\subsection{Flame "puffing"}

As mentioned in Section 3.1, flame "puffing" events were recorded occasionally at the current operating condition. To identify such events in a systematic way, spatial integration was performed on instantaneous $\mathrm{OH}^{*}$ signal from the bottom section of the combustor (where blow-out was observed). As an example, a segment of the resulting time-dependent $\left\langle\mathrm{OH}^{*}\right\rangle$ (normalized by its time mean $\left.\overline{\left\langle\mathrm{OH}^{*}\right\rangle}\right)$ is shown in Fig $\left\{5\right.$ b. At around $t=0.15 \mathrm{~s},\left\langle\mathrm{OH}^{*}\right\rangle$ drops to nearly zero, indicating a flame blow-out in the bottom section. This is followed by a robust re-light process, which registers a substantial overshoot in $\left\langle\mathrm{OH}^{*}\right\rangle$. In comparison, in stable burning flame before and after the "puffing", $\left\langle\mathrm{OH}^{*}\right\rangle$ fluctuates relatively mildly around its mean value.

The method to correlate flame "puffing" and jet flapping is described below. Fig.5 plots the normalized temporal coefficients of the flapping mode $\left(a_{F}\right)$, extracted from simultaneously measured flow fields. Note that $a_{F}$ exhibited multiple peaks in the frequency domain (also implied from the varying its cycle duration in Fig.5 flame fluctuations in Fig,2, To identify flapping cycles, a zero-crossing detection algorithm was 


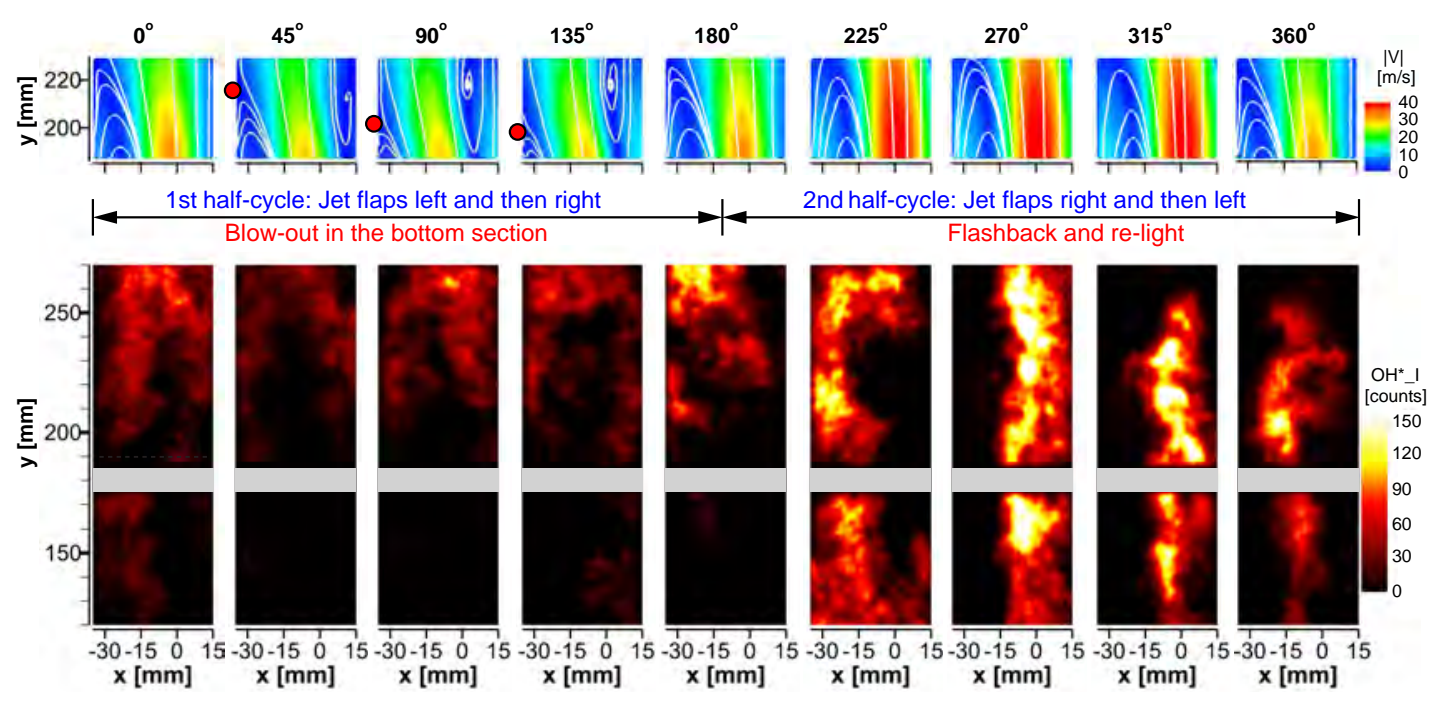

Figure 6: Top to bottom: a sequence of the instantaneous flow fields and $\mathrm{OH}^{*}$ distributions detailing a flame puffing event

used to assign $0^{\circ} / 360^{\circ}$ or $180^{\circ}$ to corresponding frames, assuming a sinusoidal behavior of $a_{F}$ (to be consistent with Fig (4). The phase angles were then extrapolated accordingly for frames in between of zero crossings. As can be seen, the entire flame "puffing" process is contained within one complete jet flapping cycle. Relating to the phase-dependent oscillations modeled in Fig, 4 , the blow-out occurs during the left-then-right half cycle of jet flapping, whereas re-light is not initiated until the subsequent half cycle of right-then-left flapping.

The detailed pattern of the flame "puffing" process recognized in Fig 5 is shown in Fig 6 with a sequence of instantaneous flow fields (top row) and $\mathrm{OH}^{*}$ distributions (bottom row). In order to focus on the global jet oscillation, the flow fields were reconstructed according to Eq.(1) with only the flapping mode to remove fine turbulent structures in each frame. Therefore, the velocity fields should only be considered as a reference to relative jet position. Instead of using time stamps, the frames were selected based on their assigned phase angles (labeled on top of each column) to better showcase a complete flapping cycle. The frames at phases $0^{\circ}, 180^{\circ}$ and $360^{\circ}$ were also marked out in Fig. 5 a to provide a time correlation.

First off, the flow fields exhibit a flapping pattern nearly identical to the low-order modeling given in Fig 4 . During the leftward jet swing from $0^{\circ}$ to $90^{\circ}$, the flame retreats downstream along the left wall until it is completely extinguished in the bottom section, corresponding to the zero intensity region of $\left\langle\mathrm{OH}^{*}\right\rangle$ in Fig.5. Additionally with the formation of the SRZ, flame is able to 
extend along this new back flow channel into the bottom section ( $90^{\circ}$ to $135^{\circ}$ ), leaving a small footprint in $\left\langle\mathrm{OH}^{*}\right\rangle$ (see Fig.5b). Then $\mathrm{OH}^{*}$ intensifies in the middle section as the jet swings right to $180^{\circ}$. In the next half-cycle, the rightward jet swing continues and opens up the back flow into the LRZ. This is accompanied by a pronounced flashback $\left(180^{\circ}\right.$ to $\left.225^{\circ}\right)$, followed immediately by a strong re-light of seemingly the entire jet at $270^{\circ}$, corresponding to the significant overshoot in Fig.5b. The subsequent leftward recovering of jet to $360^{\circ}$ causes the flame to shrink and drift away from the right wall.

\subsection{Discussion}

The primary influence of jet oscillation on combustion stability near LBO can be deduced based on the above analysis.

First, as the jet flaps, it impinges and detaches constantly from the left wall, evident from the motion of the impingement point (red mark) in both Fig. 4 a and Fig.6. This causes the back flow into the LRZ to decrease and increase (see Fig,4a). Since the LRZ is crucial for mixing of burned and unburned gas, a reduction in entrainment likely makes the LRZ less suitable for flame holding (e.g., due to a reduction in temperature) and vice versa. This could be the primary reason for flame to shrink and expand (also become more intense) during the flapping cycle, as observed in Fig. $4 \mathrm{~b}$.

Second, the results in Fig 6 suggest that flame "puffing" is triggered essentially by the same mechanism as the general flame fluctuation in Fig,4b. Following the above argument, jet impingement can result in significant build-up of unburned gas in the LRZ. In the extreme of a "puffing" event, this change in the LRZ may lead to flame quenching and eventually to blow-out in the region. As the impingement point shifts downstream, flame flashes back to the bottom section and encounters large amount of unburned fuel-air mixture, resulting in robust re-light in the bottom section.

Third, Fig 6 also shows that, although flame could extend back into the bottom section via the SRZ, this short-lived back flow channel could not result in a full re-light. On the other hand, the SRZ may play a significant role in sustaining the flame in the middle section during flame "puffing". This can be seen from the increasing $\mathrm{OH}^{*}$ intensity from $45^{\circ}$ to $90^{\circ}$. 
(a)

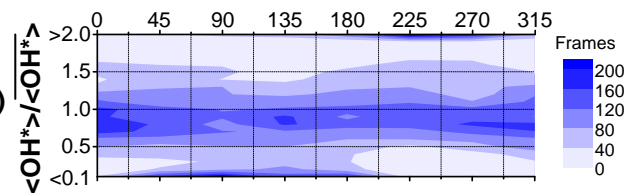

(b)

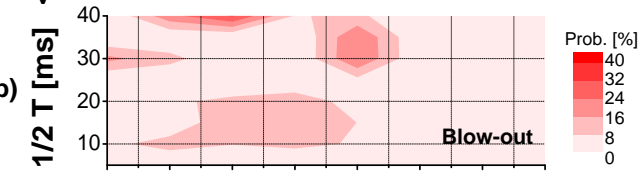

(c)

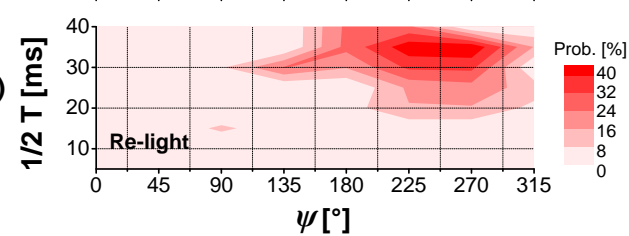

Figure 7: Statistical mapping of (a) $\left\langle\mathrm{OH}^{*}\right\rangle$ and (b-c) partial blow-out/re-light events

\section{Probability analysis of combustion instability}

One important question that still remains is whether or not other mechanisms can also result in flame "puffing". This is addressed in this section by looking for statistical correlations between jet oscillation and combustion instability. Four sets of measurements containing a total of $14 \mathrm{k}$ frames (including about 80 complete flapping cycles) were analyzed. The blow-out events were defined as frames containing $\left\langle\mathrm{OH}^{*}\right\rangle$ less than $10 \%$ of the mean value $\overline{\left\langle\mathrm{OH}^{*}\right\rangle}$. Re-light events were defined as $\left\langle\mathrm{OH}^{*}\right\rangle>2 \overline{\left\langle\mathrm{OH}^{*}\right\rangle}$. Again, only the bottom section of the combustor chamber was spatially integrated to capture flame "puffing" events. By this definition, about $20 \%$ of all the frames used were associated with flame "puffing".

Fig.7a shows a 2-D rendition of histograms of normalized $\left\langle\mathrm{OH}^{*}\right\rangle$ at different phases. Note that all the frames were sorted by their phase angles (assigned the same way as for Fig,6) into eight phase groups, each of which spanned $45^{\circ}$ and centered at the eight representative phases. As expected, the majority of the frames are found near $\overline{\left\langle\mathrm{OH}^{*}\right\rangle}$. However, the blow-out events (normalized $\left.\left\langle\mathrm{OH}^{*}\right\rangle<0.1\right)$ occur predominantly during the first half cycle of jet flapping $\left(0^{\circ}\right.$ to $180^{\circ}$ ). On the contrary, the vast majority of re-light events (normalized $\left\langle\mathrm{OH}^{*}\right\rangle>2$ ) take place during the second half cycle $\left(180^{\circ}\right.$ to $\left.360^{\circ}\right)$. This demonstrates that the pattern depicted in Fig 6 is by far the most dominant mechanism for flame "puffing".

From Fig.5, the blow-out event seems to coincide with the longest half cycle in the time-segment. In fact, a correlation was found between the pace of jet flapping and flame susceptibility to partial 
blow-out, as described in the following. The frames included in Fig.7a were further categorized by the duration of a half-cycle, 1/2T, as labeled in Fig.5. Note that an identical value was assigned to all frames belonging to the same half cycle. Analogues to the phase grouping above, the frames were also sorted into eight $1 / 2 \mathrm{~T}$ groups, centered from $5 \mathrm{~ms}$ to $40 \mathrm{~ms}$, each with a $5 \mathrm{~ms}$ span. Essentially all frames were arranged into an $8 \times 8$ matrix. Then the number of frames with partial blow-out and re-light from each cell of the matrix was divided by the total number of frames in the same cell, to derive the probability of their occurrences at a given phase and $1 / 2 \mathrm{~T}$ combination. Two probability maps were generated respectively for the blow-out and re-light events, as shown Fig.7b and c. Aside from confirming again the preferred half cycle for blow-out and re-light, flame "puffing" events are found to have much higher probability when jet flapping takes a slow pace $(1 / 2 \mathrm{~T}>30 \mathrm{~ms})$. This can be explained considering that, during a slow leftward flapping, the jet would linger at the impingement position that reduces the entrainment of burned gas into the LRZ, making the flame more susceptible to blow out in the bottom section of the chamber. On the contrary, faster-paced jet flapping is expected to be more resilient to flame "puffing". This is supported by the fact that both blow-out and re-light hardly occur at $1 / 2 \mathrm{~T}<20 \mathrm{~ms}$.

\section{Conclusions}

Combustion instabilities in a confined, premixed methane-air turbulent flame near its lean blow-out limit were examined using simultaneous particle imaging velocimetry (PIV), planar laserinduced fluorescence of $\mathrm{OH}$ radicals $(\mathrm{OH} \mathrm{PLIF})$, and $\mathrm{OH}$ chemiluminescence imaging at $5-\mathrm{kHz}$ repetition rate. Self-sustained jet oscillation within the combustor chamber was revealed by proper orthogonal decomposition (POD) of the measured velocity fields. The influence of jet oscillation on flame stabilization was analyzed by deriving its extended POD modes for the measured OH and $\mathrm{OH}^{*}$ distributions. Low-order modeling based on these spatial modes demonstrated a pronounced flame fluctuation during a jet oscillation cycle. Flame was seen to contract following the leftward swing of the jet, whereas the intensity and volume of the flame increase significantly as the jet reverses course.

An extreme case (flame "puffing") of such fluctuation was found to result in flame blow-out in the bottom section of the combustor during the first half cycle of jet oscillation. This was attributed 
to jet impingement on the left wall, limiting the entrainment of hot burned gas into the lateral recirculation zone (LRZ). However, flame was always able to restabilize with a strong re-light of the bottom section, following jet detachment and flame flashback from the middle section in the subsequent half cycle of jet flapping. This trend of blow-out followed by a re-light was demonstrated statistically as the most prevalent pattern for flame puffing to occur. In addition, slow-paced jet flapping (with a half cycle larger than $30 \mathrm{~ms}$ ) was found as more likely to trigger flame puffing.

\section{Acknowledgment}

The authors like to thank Oliver Lammel and Michael Stöhr for their technical support. Zhiyao Yin acknowledges the financial support within the Helmholtz Postdoc Programme (Grant PD-112).

\section{References}

[1] J. Wünning, J. Wünning, Prog. Energy Combust. Sci. 23 (1997) 81-94.

[2] A. Cavaliere, M. de Joannon, Prog. Energy Combust. Sci. 30 (2004) 329-366.

[3] R. Lückerath, W. Meier, M. Aigner, J. Eng. Gas Turbines Power 130 (2008) 011505.

[4] O. Lammel, H. Schütz, G. Schmitz, R. Lückerath, M. Stöhr, B. Noll, M. Aigner, M. Hase, W. Krebs, J. Eng. Gas Turbines Power 132 (2010) 121503.

[5] O. Lammel, M. Stöhr, P. Kutne, C. Dem, W. Meier, M. Aigner, J. Eng. Gas Turbines Power 134 (2012) 041506.

[6] H. Schütz, R. Lückerath, B. Noll, M. Aigner, Int. J. Energy Clean Env. 8 (2007).

[7] Z. Yin, P. Nau, I. Boxx, W. Meier, in: Proc. ASME Turbo Expo 2015, Power for Land, Sea and Air, June 15-19, Montreal, Canada.

[8] Z. Yin, I. Boxx, M. Stöhr, O. Lammel, W. Meier, in: AIAA Scitech 2016, Jan 4-7, San Diego, California.

[9] A. Maurel, P. Ern, B. Zielinska, J. Wesfreid, Phys. Rev. E 54 (1996) 3643. 
[10] G. Nathan, S. Hill, R. Luxton, J. Fluid Mech. 370 (1998) 347-380.

[11] J. Mi, G. Nathan, R. Luxton, Flow Turbul. Combust 67 (2001) 1-23.

[12] N. Lawson, M. Davidson, J. Fluids Struct. 15 (2001) 59-81.

[13] A. Mataoui, R. Schiestel, J. Fluids Struct. 25 (2009) 60-79.

[14] O. Semeraro, G. Bellani, F. Lundell, Exp. Fluids 53 (2012) 1203-1220.

[15] G. Cafiero, G. Ceglia, S. Discetti, S. Ianiro, T. Astarita, G. Cardone, in: PIV13, Delft, The Netherlands, July 1-3, 2013.

[16] G. Nathan, J. Mi, Z. Alwahabi, G. Newbold, D. Nobes, Prog. Energy Combust. Sci. 32 (2006) $496-538$.

[17] G. Berkooz, P. Holmes, J. L. Lumley, Annu. Rev. Fluid Mech. 25 (1993) 539-575.

[18] M. Stöhr, R. Sadanandan, W. Meier, Exp. Fluids 51 (2011) 1153-1167.

[19] L. Sirovich, Q. Appl. Math. 45 (1987) 561-571.

[20] J. Borée, Exp. Fluids 35 (2003) 188-192. 